EPJ Web of Conferences 113, 06008 (2016)

DOI: $10.1051 /$ epjconf/201611306008

CC Owned by the authors, published by EDP Sciences, 2016

\title{
Pairing and quartetting in medium mass nuclei
}

\author{
M. Assié ${ }^{1, a}$, B. Le Crom ${ }^{1}$, L. Lefebvre ${ }^{1}$, and J.-A. Scarpaci ${ }^{2}$ \\ ${ }^{1}$ Institut de Physique Nucléaire, Université Paris-Sud-11 - CNRS/IN2P3, 91406 Orsay, France \\ ${ }^{2}$ CSNSM, Université Paris-Sud-11 CNRS/IN2P3 91405 Orsay-Campus France.
}

\begin{abstract}
Pairing and quartetting are competing phenomena in nuclei. In the selfconjugate nuclei from the $f p$-shell, the competition between $\mathrm{T}=0$ pairing (deuteron-like) and alpha clusterisation is predicted to be affected by the spin-orbit interaction. In order to probe the $\alpha$ structure of $f p$-shell nuclei, the nuclear break-up of ${ }^{40} \mathrm{Ca}$ and ${ }^{40} \mathrm{Ar}$ has been measured at GANIL. The $\alpha$ spectroscopic factors obtained for the $\mathrm{N}=\mathrm{Z}$ nucleus and the $\mathrm{N} \neq \mathrm{Z}$ nuclei do not reflect any difference. This might be an indication that the tail of the wave function in not sensitive to nuclear asymmetry. Perspectives for probing the deuteron-like pairing $(\mathrm{T}=0)$ are given.
\end{abstract}

\section{Introduction}

Pairing is a well know feature of the nuclear interaction. Beyond the mean field picture, including like-particle pairing describes nuclear structure effects such as the odd-even mass staggering. The Wigner term added to the mass formula could be a signature of the $\mathrm{T}=0$ pairing which is specific to neutron-proton pairing. Like-particle pairing in the $\mathrm{T}=1$ channel can be accounted for at normal density in the BCS approach by weakly coupled Cooper pairs.

Infinite nuclear matter BCS calculations in ref.[1] give the evolution of the size of the Cooper pair as a function of the density. For the low density region, the size of the Cooper pair is smaller than the inter-nucleon distance. This reduced size of the pair is the signature for condensation. The best candidates to observe such phenomenon in nuclei is the halo of light nuclei where the density can reach very low values. The size of neutron-proton pairs (see ref.[2]) and quartets (two neutrons and two protons) (see ref.[3]) can also be determined in such calculations. The condensation phenomenon can be observed at normal density for deuteron-like pairs and at lower density for alpha-like particles. So that in infinite matter, the deuteron condensation prevails over alpha condensation. In nuclei, the picture may change due to the effect of the spin-orbit interaction.

Indeed, the spin-orbit force is affecting the neutron-proton interaction, particularly in the $\mathrm{T}=0$ channel. Shell model calculations [4] and quartets model [5] show that adding the spin-orbit interaction hinders neutron-proton pairing for nuclei in the $f p$-shell. So that the best candidates for the search for neutron-proton pairing are the self-conjugate $s d$-shell nuclei. On the other hand, $\alpha$ clusterization might be stronger in the $f p$-shell nuclei.

In order to investigate such phenomena, $f p$-shell nuclei have been studied through nuclear breakup. The emission of $\alpha$ particles from ${ }^{40} \mathrm{Ca}$ and ${ }^{40} \mathrm{Ar}$ has been measured in order to investigate the

ae-mail: assie@ipno.in2p3.fr 
alpha structure of their ground state. Selected results from both studies will be presented in the two following sections.

\section{Simultaneous study of alpha break-up from the $\mathrm{N}=\mathrm{Z}$ nucleus ${ }^{40} \mathrm{Ca}$ and from the $\mathrm{N} \neq \mathrm{Z}$ nucleus ${ }^{40} \mathrm{Ar}$}

Alpha clusterisation is a well known phenomenon in excited states of nuclei close to the decay threshold as depicted in the Ikeda diagram. Very little is known about clusterisation in the ground state of nuclei. Nuclei which have a pronounced $\alpha$-cluster structure in their ground states are for example ${ }^{8} \mathrm{Be},{ }^{9} \mathrm{Be},{ }^{6} \mathrm{Li}$ and ${ }^{7} \mathrm{Li} .{ }^{8} \mathrm{Be}$ is formed out of two almost free $\alpha$-particle, roughly $4 \mathrm{fm}$ apart and barely touching one another. The two $\alpha$ particle are only held together via the Coulomb barrier. Because of the large distance between the two $\alpha$ particles, the $0^{+}$ground state of ${ }^{8} \mathrm{Be}$ has a spherical density distribution close to one third of the saturation density. The $\alpha$ structure of the ground state of heavier nuclei would give an indication of quartetting at normal density.

The existence of $\alpha$ particles into the ground state of heavier nuclei such as ${ }^{40} \mathrm{Ca}$ has been investigated. As a comparison we have measured simultaneously the nuclear break-up of ${ }^{40} \mathrm{Ar}$ which is slightly isopsin asymmetric. We have performed the measurement of nuclear break-up of ${ }^{40} \mathrm{Ar}$ beam on a ${ }^{40} \mathrm{Ca}$ target at $35 \mathrm{~A} \mathrm{MeV}$ at GANIL. The set-up was using the SPEG spectrometer coupled with the MUST2 telescopes composed double sided Silicon detectors coupled to CsI detectors (see fig.1). The excitation energy resolution obtained with this set-up was of about $500 \mathrm{keV}$ (FWHM).

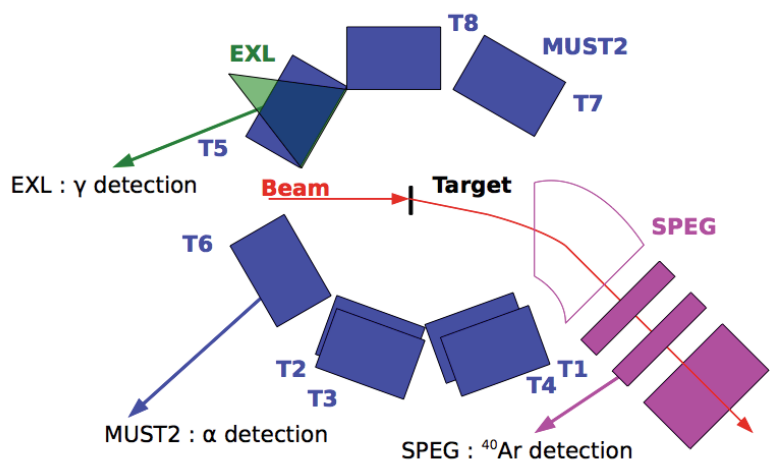

Figure 1. Experimental set-up on the SPEG line. The MUST2 Silicon detectors coupled to CsI were surrounding the ${ }^{40} \mathrm{Ca}$ target.

As for the nuclear break-up of ${ }^{40} \mathrm{Ca}$, the excitation energy spectra of ${ }^{36} \mathrm{Ar}$ obtained by missing mass method is shown in fig.2. In the laboratory frame in backward direction, the statistical decay of the target gives the main contribution whereas in the forward direction, 3 peaks can clearly be seen. They can be attributed to the first $0^{+}, 2^{+}$and $4^{+}$states of ${ }^{36} \mathrm{Ar}$ although the assignment of the $4^{+}$is more difficult (see spectrum of fig.2). The angular distribution extracted for the contributions of these three peaks were compared to a Time Dependent Schrödinger Equation (TDSE) calculation in order to extract the alpha spectroscopic factor of the ground state of ${ }^{40} \mathrm{Ca}$ (for more detail see ref.[6]). The TDSE calculations relies on the Buck potential [7] to reproduce the rotational bands in ${ }^{40} \mathrm{Ca}$ and the Pauli principle is accounted for by taking into account only the $6 \mathrm{~s}, 5 \mathrm{~d}, 4 \mathrm{~g}$ waves. 


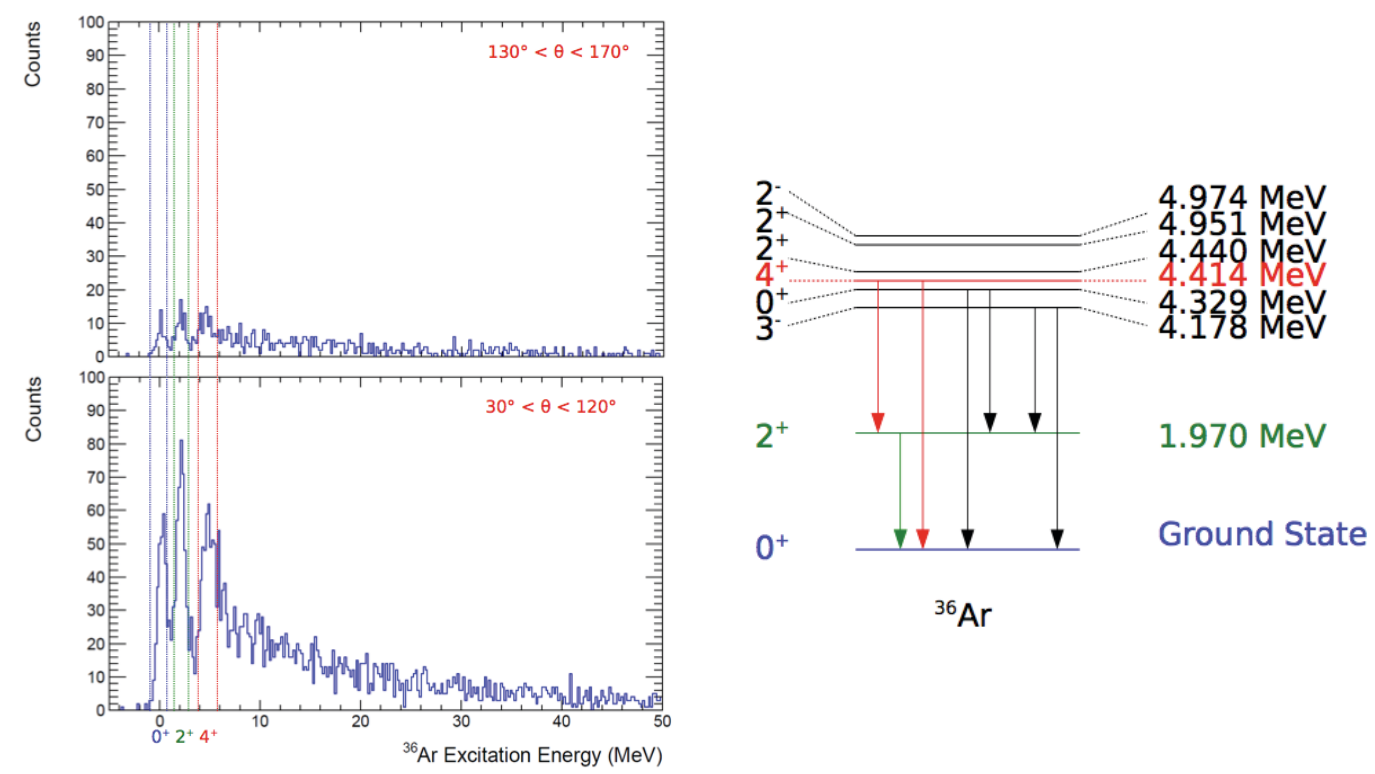

Figure 2. (Left) Missing energy spectrum of ${ }^{36} \mathrm{Ar}$ for an ${ }^{40} \mathrm{Ca}$ excitation energy range greater than $15 \mathrm{MeV}$. (Bottom) For $\alpha$-particles detected between $30 \mathrm{deg}$. and $120 \mathrm{deg}$. in the laboratory system. (Top) For $\alpha$-particles detected between $130 \mathrm{deg}$. and $170 \mathrm{deg}$. in the laboratory system. The blue gate represents the $0^{+}$ground state at $142 \mathrm{keV}$, the green one the $2^{+}$excited state at $2.1 \mathrm{MeV}$ and the red one the $4^{+}$state around $4.75 \mathrm{MeV}$. (Right) Level scheme of ${ }^{36} \mathrm{Ar}$.

The inclusive spectroscopic factor in the ground state of ${ }^{40} \mathrm{Ca}$ is found to be $3.9 \pm 0.7$. This is in good agreement with the shell model calculations from ref.[8] but much lower than the transfer reaction ${ }^{40} \mathrm{Ca}\left(\mathrm{d},{ }^{6} \mathrm{Li}\right)$ measurement from ref.[9].

The nuclear break-up of ${ }^{40} \mathrm{Ar}$ can be identified clearly in the spectra of parallel versus perpendicular momentum obtained in coincidence with ${ }^{36} \mathrm{~S}$ in SPEG (see fig.3). A contribution from the statistical decay of the target can also be seen in all direction, whereas the breakup is located in the forward left side of the beam. The angular distribution obtained in this case are shown together with the theoretical angular distributions obtained from the TDSE calculations. Assuming the same contribution for the three waves $(6 \mathrm{~s}, 5 \mathrm{~d}, 4 \mathrm{~g})$, an inclusive spectroscopic factor for $\alpha$ clusterisation in ${ }^{40} \mathrm{Ar}$ of $4.0 \pm 2.5$ is obtained.

The $\alpha$ spectroscopic factors obtained in a single measurement for ${ }^{40} \mathrm{Ca}$ and ${ }^{40} \mathrm{Ar}$ are compatible within the error bars. Nuclear break-up is probing the peripheral part of the wave function which is probably not very sensitive to the slight asymmetry between these two nuclei.

\section{Perspectives}

In order to further investigate the relationship between deuteron-like correlations and $\alpha$-like correlation, transfer reactions like $\left(\mathrm{p},{ }^{3} \mathrm{He}\right)$ which allows for deuteron-like transfer $(\Delta \mathrm{T}=0)$ but also $\Delta \mathrm{T}=1$ transfer are an efficient probe [10]. An experiment has been performed at GANIL to measure (p, $\left.{ }^{3} \mathrm{He}\right)$ and $(\mathrm{d}, \alpha)$ reaction on the closed shell nucleus ${ }^{56} \mathrm{Ni}$ and on the open shell nucleus ${ }^{52} \mathrm{Fe}$. By comparing 

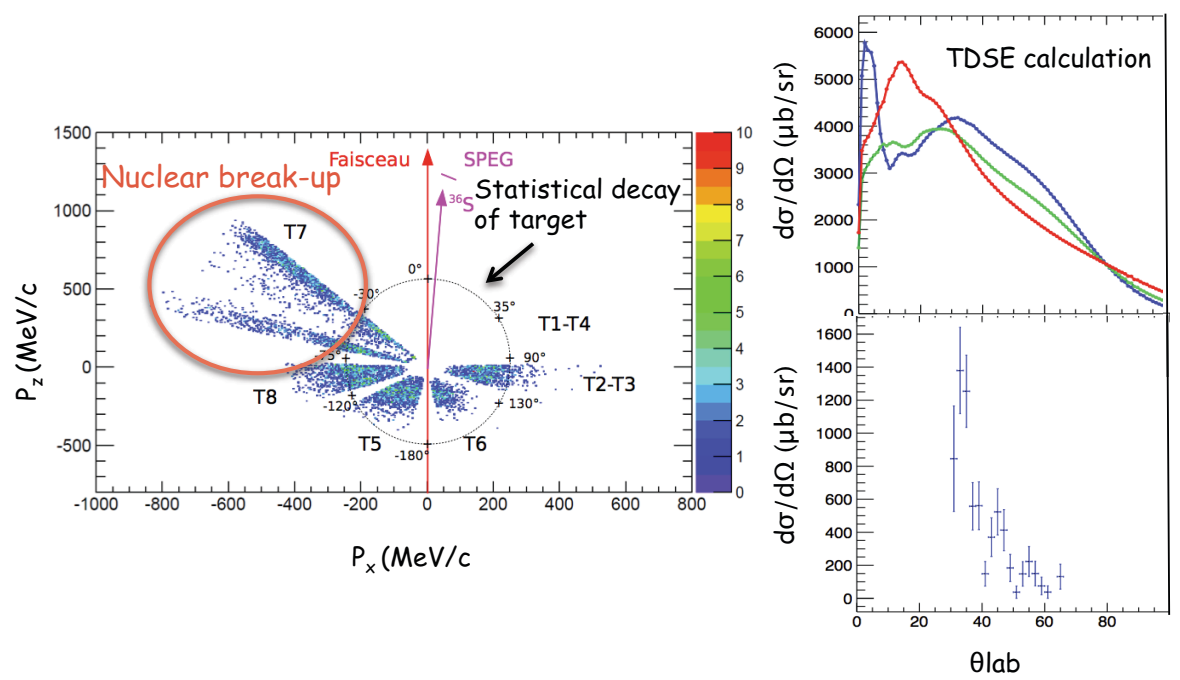

Figure 3. (Left) Parallel momentum distribution versus perpendicular momentum distribution. (Right) Angular distributions: (top) from TDSE calculations for the three contributing waves $6 \mathrm{~s}, 5 \mathrm{~d}, 4 \mathrm{~g}$ (bottom) from the experiment.

the population of the $\mathrm{T}=0$ and $\mathrm{T}=1$ states in the residual nucleus, the relative strength of the two pairing channels can be inferred. The analysis of these data is underway. They will extend the systematics of deuteron transfer reactions to the $f p$-shell region where no data are available yet. The comparison between $\alpha$ and deuteron clusterisation will be given by the comparison of $(\mathrm{d}, \alpha)$ and $\left(\mathrm{d},{ }^{6} \mathrm{Li}\right)$ transfer reactions. It will open new perspectives to the understanding of self-conjugate nuclei.

\section{References}

[1] M. Matsuo, Phys. Rev. C 73, 044309 (2006).

[2] U. Lombardo and P. Schuck, Phys. Rev. C 63038201 (2001).

[3] G. Röpke and A Schnell and P. Schuck, Phys. Rev. Lett. 80, 3177 (1998).

[4] A. Gezerlis and G.F. Bertsch and Y.L. Luo, Phys. Rev. Lett. 106, 252502 (2011).

[5] M. Sambataro and N. Sandulescu, Phys. Rev. C 91064318 (2015).

[6] L. Lefebvre, Proc. of Science (Bormio 2013) 069.

[7] B. Buck et al, Phys. Rev. C 51, 1840 (1995).

[8] W. Chung et al, Phys. Lett. B 79, 381 (1978).

[9] K. Umeda et al, Nucl. Phys. A 42988 (1984).

[10] P. van Isäcker, D.D. Warner and A. Franck, Phys. Rev. Lett. 94, 162502 (2005). 